EPJ Web of Conferences 92,02058 (2015)

DOI: $10.1051 /$ epjconf/ 20159202058

C Owned by the authors, published by EDP Sciences, 2015

\title{
Design of $h$-Darrieus vertical axis wind turbine
}

\author{
Teresa Parra $^{\mathrm{a} 1}$, Carmen Vega ${ }^{2}$, A. Gallegos ${ }^{3}$, N. C. Uzarraga ${ }^{4}$, F. Castro $^{1}$ \\ ${ }^{1}$ University of Valladolid, Spain. \\ ${ }^{2}$ University of Zulia, Venezuela. \\ ${ }^{3}$ University of Guanajuato, Mexico. \\ ${ }^{4}$ Technological University of Durango, Mexico.
}

\begin{abstract}
Numerical simulation is used to predict the performance of a Vertical Axis Wind Turbine (VAWT) H-Darrieus. The rotor consists of three straight blades with shape of aerofoil of the NACA family attached to a rotating vertical shaft. The influence of the solidity is tested to get design tendencies. The mesh has two fluid volumes: one sliding mesh for the rotor where the rotation velocity is established while the other is the environment of the rotor. Bearing in mind the overall flow is characterized by important secondary flows, the turbulence model selected was realizable $\mathrm{k}$-epsilon with non-equilibrium wall functions. Conservation equations were solved with a Third-Order Muscl scheme using SIMPLE to couple pressure and velocity. During VAWT operation, the performance depends mainly on the relative motion of the rotating blade and has a fundamental period which depends both on the rate of rotation and the number of blades. The transient study is necessary to characterise the hysteresis phenomenon. Hence, more than six revolutions get the periodic behaviour. Instantaneous flows provide insight about wake structure interaction. Time averaged parameters let obtain the characteristic curves of power coefficient.
\end{abstract}

\section{Introduction}

This research line has been developed in collaboration with four universities: University of Valladolid in Spain, University of Zulia in Venezuela and Universities of Durango and Guanajuato in Mexico.

So far it is basic research of the performance of vertical axis wind turbine to get an insight of the flow patterns and establish the methodology.

In the middle term, it is intended to propose a feasible turbine under the criteria of simple manufacture, low cost of production, easy maintenance. The idea is supplying the domestic electricity of isolated zones in developing countries.

The main advantages of VAWT are that there is no need of orientation system on the wind direction and they can operate with relatively low wind velocity. Obviously it is not suitable for large electricity production but this is not the specific purpose.

The main problems are the self starting and their low efficiency. These aspects offer the chance of further developing this technology [1]. The kind of device is an H-Darrieus, see figure 1a. It is composed by 3 blades without any torsion. This offers the possibility to apply the hypothesis of $2 \mathrm{D}$ models [2], figure $1 \mathrm{~b}$. Then the models will reproduce the flow pattern in the middle plane of the rotor. No pitch angle control system is used to fulfill the simplicity criterion.

a)

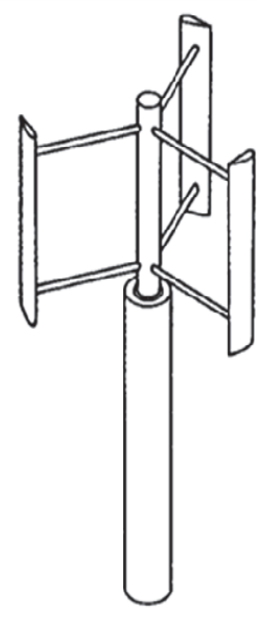

b)

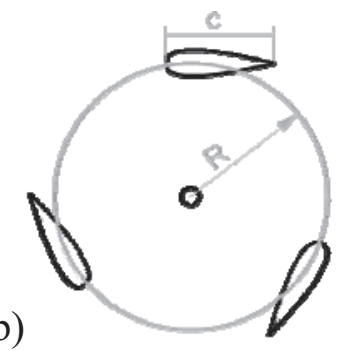

Figure 1. Set up of the H-Darrieus. a) 3D scheme. b) 2D approach.

\footnotetext{
${ }^{a}$ Corresponding author: terpar@eii.uva.es
} 
The geometrical details of a scaled down model for laboratory purposes are presented in table 1 . Then the chord has only $5 \mathrm{~cm}$ and the length $20 \mathrm{~cm}$. The airfoils belong to the family NACA. Non symmetric airfoils with no pitch control seem to show worse performance than the symmetric airfoils, [3]. The symmetric airfoil is the NACA0025 and different solidity degrees between 1 and 0.3 are used. Solidities higher than 1 are too high to be used on electricity production because of the low rotation speeds [4].

This work presents a review of the numerical details, procedure to obtain the characteristic curves, the flow patterns and tendencies when using very low solidity coefficients.

Table 1. Summary of geometrical details and the inlet conditions for the nozzles.

\begin{tabular}{|c|c|}
\hline Airfoil & NACA 0025 \\
\hline Shaft Radius & $0.01 \mathrm{~m}$ \\
\hline Rotor Radius (R) & $0.071-0.14-0.24 \mathrm{~m}$ \\
\hline Length (b) & $0.2 \mathrm{~m}$ \\
\hline Chord (c) & $0.05 \mathrm{~m}$ \\
\hline Area (A) & $0.02864 \mathrm{~m}^{2}$ \\
\hline Number of Blades (N) & 3 \\
\hline Solidity $(\sigma)$ & $1.0-0.5-0.3$ \\
\hline
\end{tabular}

\section{Numerical Model}

Sensibility to the mesh for different meshes leads to a compromise on computational cost and accuracy, concluding the 2D mesh have around 100 thousand cells.

The computational domain has two zones, see figure 2 , one in the rotor where moving reference mesh is used to establish a specific rotation velocity for the rotor. The environment has lower mesh resolution.

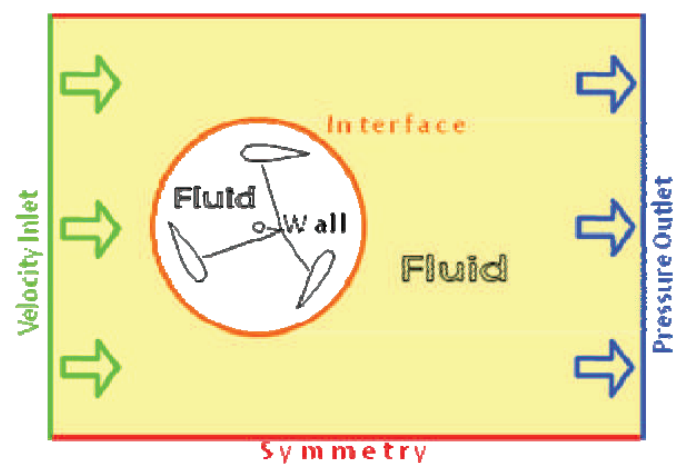

Figure 2. Computational domain and boundary conditions

Because of the moving reference mesh, the reference system is no inertial and the momentum conservation equations have two terms associated to the centrifugal and Coriolis forces.
The criteria to select the most appropriated scheme of resolution was the minimum residual on mass, lower variance of momentum coefficient and lower number of iterations per time step. To sum up, the $3^{\text {er }}$ order scheme was the most suitable.

Turbulence models of 1 and 2 equations were tested and the realizable $\mathrm{k} \varepsilon$ was the one to show better numerical stability.

Treatment near the walls was non equilibrium wall functions because boundary layer is separated from the airfoils and there are many secondary flows. In fact the wake controls the performance of the turbine.

The problem is periodic, then the time step is fixed so one revolution of the rotor takes place every 300 time steps.

The conservation equations for incompressible, transient and turbulent flows are in equations 1 to 4 .

$$
\begin{gathered}
\frac{\partial}{\partial x_{i}}\left(\rho \bar{u}_{i}\right)=0 \\
\frac{\partial}{\partial t}\left(\rho \bar{u}_{i}\right)+\frac{\partial}{\partial x_{j}}\left(\rho \overline{u_{i} u_{j}}\right)+\rho\left(2 \vec{\omega} \times \bar{u}_{i}+\vec{\omega} \times \vec{\omega} \times \vec{r}\right)=-\frac{\partial \bar{p}}{\partial x_{i}}+\frac{\partial\left(\overline{\bar{\tau}_{i j}}\right)_{e f f}}{\partial x_{j}} \\
\frac{\partial}{\partial t}(\rho k)+\frac{\partial}{\partial x_{i}}\left(\rho k u_{i}\right)=\frac{\partial}{\partial x_{j}}\left(\alpha_{k} \mu_{e f f} \frac{\partial k}{\partial x_{j}}\right)+G_{k}-\rho \varepsilon \\
\frac{\partial}{\partial t}(\rho \varepsilon)+\frac{\partial}{\partial x_{i}}\left(\rho \varepsilon u_{i}\right)=\frac{\partial}{\partial x_{j}}\left(\alpha_{\varepsilon} \mu_{e f f} \frac{\partial \varepsilon}{\partial x_{j}}\right)+C_{1 \varepsilon} \frac{\varepsilon}{k} G_{k}-C_{2 \varepsilon} \rho \frac{\varepsilon^{2}}{k}
\end{gathered}
$$

where $\rho$ is density, $\omega$ is the angular velocity of the rotor, $k$ is the turbulence kinetic energy and $\varepsilon$ is its dissipation.

\section{Prediction of Power Curves}

After full convergence is achieved, the flow is periodic. At low rotation velocities the convergence request more than 10 revolutions but at high rotation velocities, 4 revolutions are enough.

In the figure $3 \mathrm{a}$ the periodic behaviour of instantaneous momentum coefficient versus the angle of one airfoil is shown. When the momentum coefficient is time averaged during one revolution of the rotor, it is obtained one point of the curve momentum coefficient versus the tip speed ratio (ratio of velocity of the trailing point of the blade versus the velocity of the wing, TSR), figure $3 \mathrm{~b}$. When changing the rotation velocity of the rotor, different points of the curves are calculated to get the whole curve.

Multiplying the momentum coefficient by the tip speed ratio, the power coefficient is obtained in the figure $3 \mathrm{c}$. This is a measure of the efficiency, having in mind the maximum comes from the ideal theory of Betz (59\%). It is not possible to take the $100 \%$ of kinetic energy of the wind and bring it to stagnation because it would be against mass conservation equations. Design conditions are related with the peak of pressure coefficients $\mathrm{C}_{\mathrm{p}, \max }=0.303$ and $\mathrm{TSR}=1.023$ 


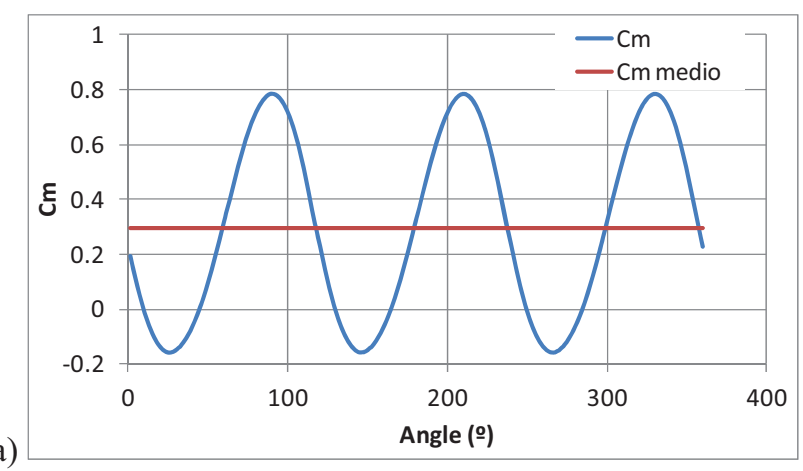

a)

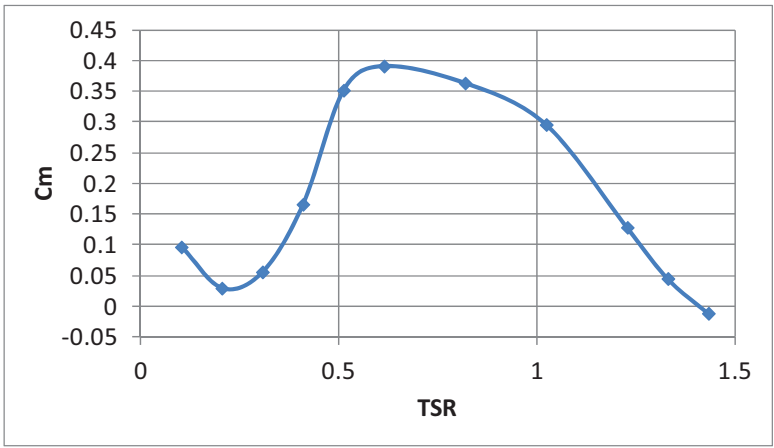

b)

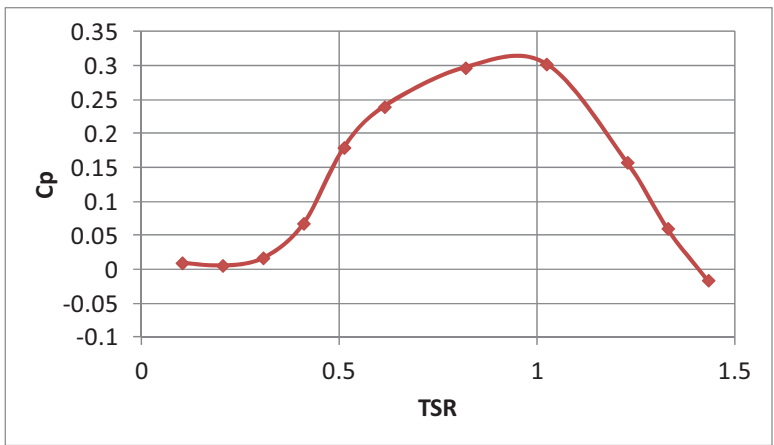

Figure 3. NACA0025, solidity 1 a) Instantaneous momentum coefficient b) Averaged momentum coefficient versus TSR c) Averaged power coefficient versus TSR.

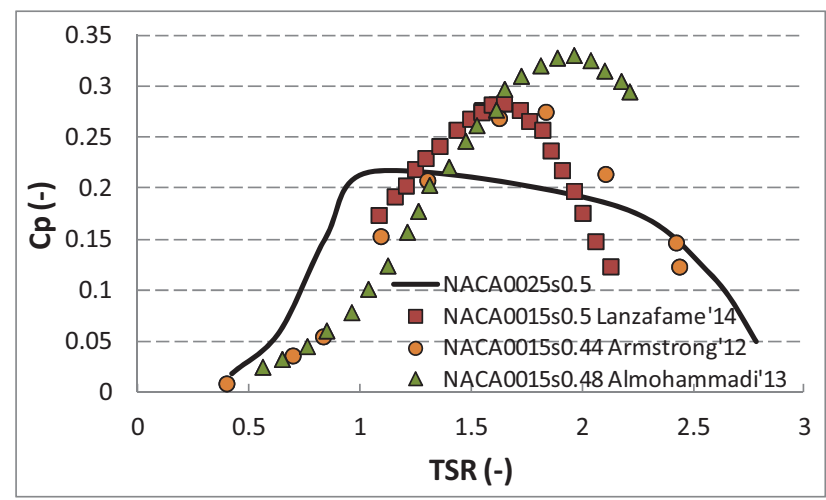

Figure 4. Averaged power coefficient versus the Tip Speed Ratio for NACA 0025, solidity 0.5. Experimental results of similar solidity and airfoils NACA0015 ref. $[6,7,8]$.

Figure 4 shows the numerical power curve for a rotor of solidity 0.5 , represented in solid line, as well as experimental measurements from other sources for rotors with similar solidity and foils NACA0015. Even though predicted range of operating Tip Speed Ratios of power generation agrees with the experimental ones, the numerical model under predicts the experimental peak power coefficient.

\section{Flow Pattern}

If angle $0^{\circ}$ corresponds with noon position in the clock and the azimuthal coordinate increases counterclockwise, minimum instantaneous power coefficient corresponds approximately with positions of the blades 0, 120240 degrees whereas maximum instantaneous power coefficient that corresponds with blades located near 60 180 and 300 .

Figure 5 has two slices with vorticity contours for the minimum and maximum instantaneous power coefficient respectively. Vorticity let identify the vortex shedding of the wake. It is clear the blade at 0 degrees receives perturbed flow by the wake from blade at 120 degrees.

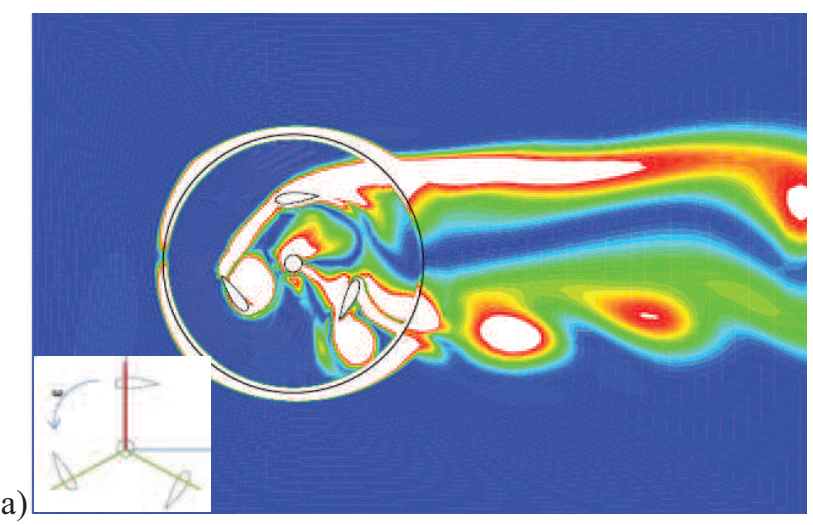

b)

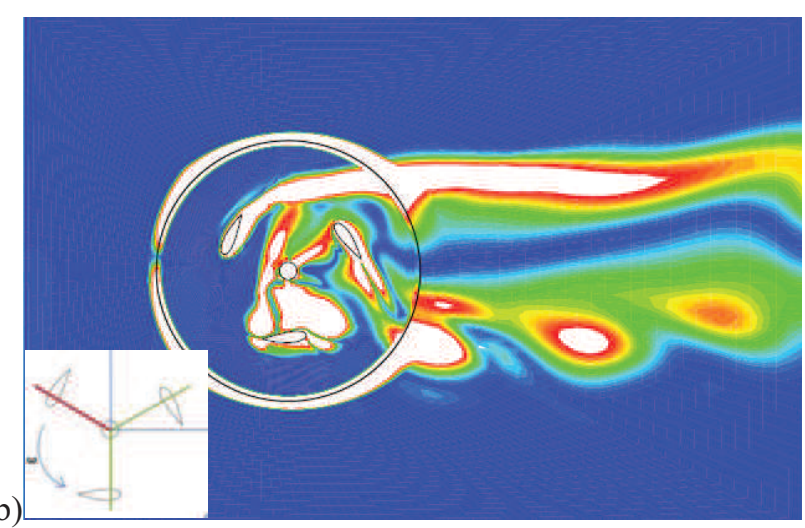

Figure 5. Contours of vorticity NACA 0025 for an incident wind speed of $7 \mathrm{~m} / \mathrm{s}$ and solidity 1 at design conditions. a) at minimum instantaneous power coefficient $b$ ) at maximum instantaneous power coefficient.

\section{Influence of solidity}

The change the solidity of the rotor is achieved by modifying the rotor diameter.

The reduction of the solidity increases the range of TSR operating as turbine (positive power coefficient) and reduces the maximum power coefficient, see figure 6 and table 2. These results agree with literature findings [9]. 


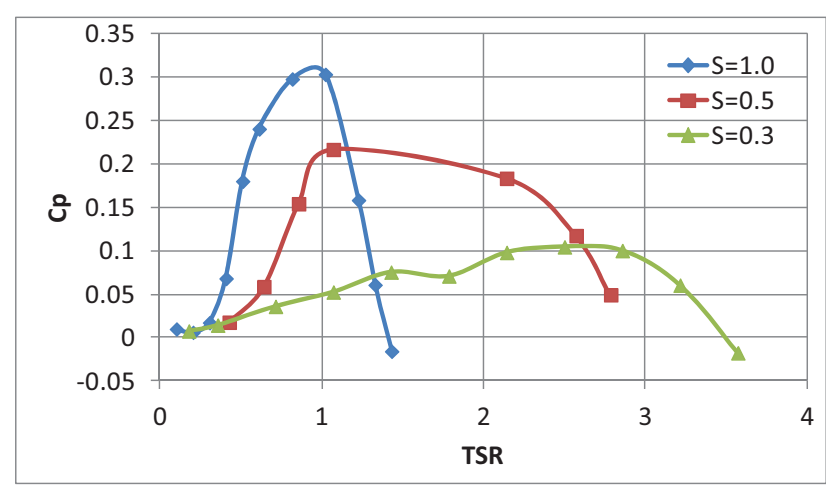

Figure 6. Averaged power coefficient versus the Tip Speed Ratio on NACA 0025 for an incident wind speed of $7 \mathrm{~m} / \mathrm{s}$ and solidities $1,0.5$ and 0.3 .

Table 2. Influence of solidity on performance of NACA0025.

\begin{tabular}{|c|c|c|c|}
\hline Solidity & $\mathbf{1 . 0}$ & $\mathbf{0 . 5}$ & $\mathbf{0 . 3}$ \\
\hline Range of TSR & $0.204-$ & $0.429-$ & $0.357-$ \\
& 1.330 & 2.789 & 3.571 \\
\hline C p, max & 0.303 & 0.216 & 0.104 \\
\hline $\begin{array}{c}\text { TSR at peak } \\
\text { power coef. }\end{array}$ & 1.023 & 1.071 & 2.500 \\
\hline
\end{tabular}

The reduction of power coefficient is compensated with the increase of the rotor radius. Hence, the power generated is increasing while reducing the solidity. Table 3 shows power production for an H-Darrieus scaled up with proportion 1:10. The dimensions and range of power correspond with those of the Aeolos-V 300w vertical wind turbine [10].

Table 3. Sensibility analysis for power production at scale 1:10.

\begin{tabular}{|c|c|c|c|c|}
\hline & $\mathbf{C}_{\mathbf{p}, \max }$ & $\boldsymbol{T S R}_{\text {design }}$ & $\begin{array}{c}\boldsymbol{\Omega}_{\text {design }} \\
(\mathbf{r a d} / \mathbf{s})\end{array}$ & $\begin{array}{c}\text { Power } \\
\text { (W) }\end{array}$ \\
\hline $\boldsymbol{\sigma}=\mathbf{1 . 0 4 7}$ & 0.303 & 1.023 & 10 & 182.3 \\
\hline $\boldsymbol{\sigma}=\mathbf{0 . 5}$ & 0.216 & 1.071 & 5 & 272.3 \\
\hline $\boldsymbol{\sigma}=\mathbf{0 . 3}$ & 0.104 & 2.500 & 7 & 218.5 \\
\hline
\end{tabular}

\section{Conclusions}

A methodology has been proposed to simulate numerically the performance of VAWT H-Darrieus. Performance is analyzed from the flow pattern and the characteristic curves of power coefficient versus TSR.

It was studied the influence of solidity between 1 and 0.3. Lower solidity rotors are more suitable from the aerodynamic point of view, because of the increasing range of operation of TSR.

Although lower solidity rotors are precursors of lower efficiency, the power extracted might be higher due to the increase of the rotor radius.

\section{Acknowledgment}

This is a collateral research of the project reference ENE2011-25468 from the Spanish Ministry of Science and Innovation.

\section{References}

1. J. R. Baker. Journal of Wind Engineering and Industrial Aerodynamics. Vol. 15 pp.369-380 (1983)

2. N.C. Uzarraga, A. Gallegos, M. T. Parra, J. M. Beltran. ASME ESFuelCell2012-91113 (2012)

3. T. Parra, C. Uzarraga, A. Gallegos, M.A. Rodriguez, F. Castro. FEDSM2014-21356 (2014)

4. T. Parra, M. Esteban, F. Gonzalez, C. Uzarraga, A. Gallegos, F. Castro. International Conference of Advanced Technologies and Sciences (2014)

5. X. Liu, Q. Dang, G. Xi. Engineering Applications of Computational Fluid Mechanics Vol. 2 no. 2 pp. 130140. (2008)

6. R. Lanzafame, S. Mauro, M. Messina Energy Procedia 45, pp 131 - 140 (2014)

7. S. Armstrong, A. Fiedler, S. Tullis Renewable Energy 41 pp 13-22 (2012)

8. K.M. Almohammadi, D.B. Ingham, L. Ma, M. Pourkashan Energy 58 pp 483-493(2013)

9. A.Shawn, A.Fiedler, S.Tullis. Renewable Energy, Vol. 41, pp. 13-22 (2012)

10. www.windturbinestar.com $/ 300 \mathrm{w}$-vertical-windturbine.html (last visit 31.10.2014) 